\title{
VAC_18 - Evaluation of humoral and cellular immune response after heterologous prime-boost immunization against SARS-CoV-2
}

Patrick Orestes de Azevedo ${ }^{1 *}$; Natália Satchiko Hojo-Souza ${ }^{1}$; Lídia Paula Faustino ${ }^{1}$; Beatriz Senra Álvares da Silva Santos ${ }^{1}$; Alexandre de Magalhães Vieira Machado ${ }^{1}$; Ricardo Tostes Gazzinelli ${ }^{1}$.

${ }^{1}$ Fiocruz/CPqRR.

Introduction: COVID-19 is an infectious disease caused by the coronavirus named SARS-CoV-2, which emerged in the Wuhan city (China) in December 2019. The disease spread quickly to all continents, being recognized by the World Health Organization (WHO) as a pandemic in March 2020. Given these circumstances, the production of vaccines to meet global demand may take time. In this context, Brazil will certainly need its own vaccines for COVID-19.

Objective: The present study aimed to develop a vaccine for COVID-19 using recombinant plasmids and human Adenovirus 5 (hAd5) carrying gene sequences of the RBD domain (spike protein) and the nucleocapsid protein $(\mathrm{N})$ from SARS-CoV-2.

Methodology: The recombinant plasmid and hAd5 platform were chosen for the development of a vaccine against COVID-19 considering the relative stability, easy handling, safety and immunogenicity induced by both agents. Commercial plasmids containing the gene sequences encoding RBD and N proteins from SARS-CoV-2 were digested with specific restriction enzymes and cloned into the plasmid pcDNA3 (expression plasmid). For the recombinant hAd5 production, a transfer vector (pAdCMV-Link) that carries the gene sequences of RBD and N proteins was used. In addition, the pJM17 (hAd-5 genome) presents regions of homology with pAdCMV-Link, which enables homologous recombination of plasmids and the transfer of the gene sequences encoding SARS-CoV-2 proteins to the hAd5 genome. Mice were immunized using a heterologous prime/boost protocol with plasmid DNA (100ug) as a prime, followed by a boost with hAd5 ( $\left.10^{9} \mathrm{pfu}\right)$ three weeks later, containing the RBD and $\mathrm{N}$ gene sequences. Plasmids were inoculated intramuscularly and hAd5 intranasally. The humoral immune response was assessed by measuring the levels of specific antigen IgG present in the plasma and bronchoalveolar lavage (BALF) of the immunized animals. Cellular immune response was evaluated by the ability of splenocytes to produce IFN- $\gamma$ under stimulation with recombinant proteins (RBD and $\mathrm{N}$ ).

Results: The evaluation of specific antibodies response showed a significant result for IgG titer anti-N $(\mathrm{p}<0.001)$ in plasma and BALF samples. On the other hand, cellular immune response showed significant results for both targets (RBD and N). Splenocytes from mice immunized with DNARBD/AdRBD exhibited high IFN- $\gamma$ secretion in response to specific stimulation in comparison to control groups $(p<0.01)$. Similar result was observed in splenocytes from mice immunized with DNA N/AdN ( $p<0.001)$.

Conclusion: These findings suggest that the RDB and $\mathrm{N}$ proteins vectored with plasmid and hAd5 may be interesting vaccine candidates against SARS-CoV-2. Therefore, the next step is to assess the percentage of protection after challenge with the SARS-CoV-2 in hACE transgenic mice immunized with the heterologous prime/boost protocols.

Keywords: Adenovirus; COVID-19; Proteins 